\section{Mathematical model that describes the transition from thermal to photochemical damage in retinal pigment epithelial cell culture}

\author{
Clifton D. Clark III, ${ }^{a}$ Michael L. Denton, ${ }^{\mathrm{a}}$ and \\ Robert J. Thomas ${ }^{b}$ \\ aTASC Inc., Biomedical Sciences and Technologies Department, \\ Brooks City-Base, Texas 78235 \\ bAir Force Research Laboratory, 711 HPW/RHDO, Brooks City-Base, \\ Texas 78235
}

\begin{abstract}
We propose a rate process model for describing photochemical damage to retinal cells by short wavelength laser exposures. The rate equation for photochemical damage contains a positive rate that is temperature independent, and a negative (quenching) rate that is temperature dependent. Using the traditional Arrhenius integral to describe thermal damage, we derive damage threshold doses for both thermal and photochemical mechanisms, and show that the model accounts for the sharp transition from thermal to photochemical damage thresholds that have recently been observed in an in-vitro retinal model. @ 2011 Society of Photo-Optical Instrumentation Engineers (SPIE). [DOI: 10.1117/1.3544504]
\end{abstract}

Keywords: retina damage model; thermal damage; photochemical damage.

Paper 10586LR received Oct. 29, 2010; revised manuscript received Dec. 28, 2010; accepted for publication Dec. 30, 2010; published online Feb. 17, 2011.

\section{Introduction}

For long pulse durations ( $>1 \mathrm{~s}$ ), laser induced tissue damage can be divided into two broad categories: thermal and nonthermal (photochemical). Thermal damage has been modeled by the Arrhenius damage integral, first proposed by Henriques ${ }^{1}$ in 1947, with great success. ${ }^{2-5}$ Other models have been developed to address the shortcomings of the Arrhenius integral, ${ }^{6,7}$ but it continues to be a popular method for predicting thermal damage thresholds due to its relative simplicity.

No such damage model exists for predicting photochemical damage thresholds. Perhaps the demand for such a model has been low because photochemical damage is defined by the principle of reciprocity, where the total radiant exposure remains constant with exposure duration, allowing damage to be predicted by simply considering the total energy delivered. ${ }^{8}$ However, in his in-vitro retinal model, Denton et al. showed that at $413 \mathrm{~nm}$, damage thresholds follow a thermal trend line for exposure durations up to $60 \mathrm{~s}$ and can be a factor of 2

Address all correspondence to: Robert J. Thomas, Air Force Research Laboratory, AFRL/RHDO, 2624 Louis Bauer Drive, Brooks City-Base, Texas 78235-5128. Tel: 200536 6558; Fax: 210536 6558; E-mail: Robert.Thomas@brooks.af.mil. higher than the reciprocity radiant exposure..$^{9}$ From this work, the damage threshold mechanism seems to transition sharply from thermal to photochemical somewhere between 60 and $100 \mathrm{~s}$, and a similar trend has been described in an animal model as well. ${ }^{10}$

In this paper, we propose a rate equation to describe photochemical damage that includes a temperature dependence. The model assumes the creation of some activated molecule $\left(B^{*}\right)$ through a photon absorption that is independent of temperature and a temperature dependent quenching rate that removes $B^{*}$ (Fig. 1). We treat thermal and photochemical damage mechanisms as independent processes, the damage threshold is defined as the lowest threshold of the two. The temperature independence of $B^{*}$ creation and the temperature dependence of quenching leads to a sharp cutoff for photochemical damage. Above some critical temperature, the photochemical damage mechanism is completely negated, allowing the damage threshold to be determined by thermal damage at energies much higher than the reciprocity energy.

\section{Damage Model}

\subsection{Thermal Damage Rate}

We assume thermal damage to be described by the usual Arrhenius rate equation, where some molecule $A$ (typically assumed to be a protein) is converted to a denatured state $A^{*}$. The incident light provides a heat source which raises the temperature of the cells, increasing the rate of this reaction. The damage rate is given as

$$
\frac{d\left[A^{*}\right]}{d t}=Z_{1} e^{-E_{a 1} / R T(t)}
$$

Here the brackets, $[\cdot]$, denote molar concentrations, and $R$ and $T(t)$ are the gas constant and system temperature (in Kelvin), respectively. The two constants, $E_{a 1}$ and $Z_{1}$, are termed activation energy and collision frequency. These rate coefficients must be experimentally determined, and are strongly dependent on the system and damage endpoint assessed. Several sets of coefficients exist in the literature. ${ }^{11}$

\subsection{Photochemical Damage Rate}

We propose a two-process rate equation to describe photochemical damage. Some molecule $B$ absorbs a single photon to create an activated molecule $B^{*}$. This activated molecule may then be deactivated through an interaction with some other molecule, $\xi$, provided by the cell. The activated molecule, $B^{*}$, may also initiate a chain of events that eventually leads to cell death (Fig. 1).

The total rate of creation for $B^{*}$ can then be written as a positive rate dependent only on the incident photon flux plus a negative rate that accounts for the chemical reaction-based removal of $B^{*}$,

$$
\frac{d\left[B^{*}\right]}{d t}=\epsilon \Phi[B]-Z_{2} e^{-E_{a 2}^{\prime} / R T(t)}[\xi]\left[B^{*}\right] .
$$

Here, $\Phi$ is the incident photon flux density (number of photons per area per time), $\epsilon$ denotes an efficiency factor, which is the probability that an incident photon is absorbed and creates the

1083-3668/2011/16(2)/020504/3/\$25.00 @ 2011 SPIE 


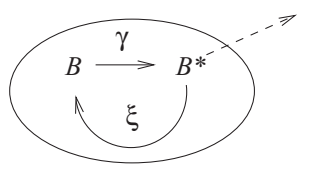

Fig. 1 Photochemical damage mechanism. A single photon $(\gamma)$ is absorbed by $B$ to produce $B^{*}$ and some other molecule $\xi$ reacts with $B^{*}$ to regenerate $B$, or a non-reactive product of $B$. Any accumulation of $B^{*}$ can be available to trigger a chain reaction resulting in cell death (dashed line)

activated molecule, $B^{*}$, and $E_{a 2}$ and $Z_{2}$ are activation energy and collision frequency associated with $\xi$ and $B^{*}$ reacting to give $B$. We will assume that the molecules $B$ and $\xi$ are abundant, so their concentrations can be considered constant. We then use the simplified rate equation for $B^{*}$

$$
\frac{d\left[B^{*}\right]}{d t}=\epsilon \Phi-Z_{2} e^{-E_{a 2} / R T(t)}\left[B^{*}\right] .
$$

We assume photochemical damage to be caused by a buildup of $B^{*}$, and so the damage threshold will correlate to a concentration of $B^{*}$. The relationships between positive and negative reaction rates, the temperature independence of damage creation, and the temperature dependence of damage quenching, are unique to the photochemical mechanism.

\section{Damage Threshold Predictions}

We seek to find an expression for the threshold radiant exposure for causing damage to cells. In our analysis here, the thermal and photochemical damage mechanisms compete. Thermal damage is caused when $A^{*}$ reaches some threshold concentration, $\left[A^{*}\right]_{\mathrm{th}}$, and photochemical damage is caused when $B^{*}$ reaches a threshold concentration, $\left[B^{*}\right]_{\text {th }}$. Both thermal and photochemical mechanisms will have a threshold radiant exposure to cause damage. The two rate equations, (1) and (3), are integrated to give $\left[A^{*}\right]$ and $\left[B^{*}\right]$ as a function of time and then solved for the radiant exposure $(\mathrm{H})$ that gives $\left[A^{*}\right]_{\mathrm{th}}$ or $\left[B^{*}\right]_{\mathrm{th}}$ for the exposure duration $\tau$. The lower of these two thresholds will be the observed damage threshold for the exposure.

In the experiments conducted by Denton et al., ${ }^{9}$ retinal pigment epithelial (RPE) cells were exposed to $413 \mathrm{~nm}$ laser radiation for durations of $0.1,1.0,20,40,60,100$, and $200 \mathrm{~s}$. For exposures greater than about $20 \mathrm{~s}$, the system reached steadystate early in the exposure, and the temperature can be assumed constant throughout. The transition from thermal to photochemical damage occurred somewhere between 60 and $100 \mathrm{~s}$, which we seek to describe, so we can assume the exposure temperature to be constant.

Due to the linearity of the heat equation, the peak steadystate temperature can be written as a linear function of the peak incident power, $E_{0}$,

$$
T=m E_{0}+T_{0},
$$

where $T_{0}$ is the initial (ambient or body) temperature. The slope of this line, $m$, will depend on the specific laser parameters and system configuration.

\subsection{Thermal Damage Threshold}

Integrating Eq. (1) with the temperature held constant gives

$$
\left[A^{*}\right]=Z_{1} e^{-E_{a 1} / R T} \tau,
$$

where $\tau$ is the exposure duration and $T$ is the exposure temperature. The threshold temperature, $T_{\mathrm{th}}$, is the temperature that leads to the threshold damage accumulation, $\left[A^{*}\right]_{\mathrm{th}}$. However, the exposure temperature is related to the exposure irradiance through Eq. (4). Inserting $T=m E_{\text {th }}+T_{0}$ into Eq. (5) and solving for $E_{t h}$ gives the damage threshold irradiance. Most rate coefficients $\left(Z_{1}\right.$ and $\left.E_{a 1}\right)$ for thermal damage are measured assuming that $\left[A^{*}\right]_{\mathrm{th}}=1$, so the damage threshold irradiance is

$$
H_{\mathrm{th}}=\tau E_{\mathrm{th}}=\frac{\tau}{m}\left[\frac{E_{a 1}}{R \ln \left(Z_{1} \tau\right)}-T_{0}\right] .
$$

\subsection{Photochemical Damage Threshold}

To integrate Eq. (3), we define $K_{2}(T) \equiv Z_{2} e^{-E_{a 2} / R T}$ and multiply by the integration factor $e^{K_{2} t}$ which gives,

$$
\left[B^{*}\right]=\frac{\epsilon \Phi}{K_{2}}\left[1-e^{-K_{2}(T) \tau}\right] .
$$

We must define $\left[B^{*}\right]_{\text {th }}$ to obtain a damage threshold exposure. For this, we turn to the principle of reciprocity. Here, the radiant exposure remains constant as the exposure duration varies. It follows that as the exposure duration increases the incident irradiance is reduced, which in turn implies that the exposure temperature is lowered. Reciprocity suggests that the quenching rate, $K_{2}(T)$, is small for low temperature rises.

We define the reciprocity radiant exposure as $H_{r}$. The photon flux density can be written in terms of the radiant exposure as $\Phi=H / \tau h v$, where $h v$ is the photon energy and $h$ is Planck's constant. At reciprocity then, Eq. (7) can be written as,

$$
\left[B^{*}\right]_{\mathrm{th}}=\frac{\epsilon}{K_{2}(T)} \frac{H_{r}}{\tau h \nu}\left[1-e^{-K_{2}(T) \tau}\right] .
$$

Since $K_{2}(T)$ is small here, we may expand the exponential in a power series and ignore all second order and higher terms $\left(\left(K_{2} \tau\right)^{2} \ll 1\right)$ and Eq. (8) simplifies to

$$
\left[B^{*}\right]_{\mathrm{th}}=\frac{\epsilon H_{r}}{h v} .
$$

Using this definition, we can solve Eq. (7) for the threshold radiant exposure,

$$
H_{\mathrm{th}}=H_{r} K_{2}(T) \tau\left[1-e^{-K_{2}(T) \tau}\right]^{-1} .
$$

This gives the damage threshold radiant exposure for some fixed temperature. However, the exposure temperature is also dependent on $H$ through Eq. (4) so Eq. (10) becomes

$$
H_{\mathrm{th}}=H_{r} K_{2}\left(m \frac{H_{\mathrm{th}}}{\tau}+T_{0}\right) \tau\left[1-e^{-K_{2}\left[m H_{\mathrm{th}} / \tau+T_{0}\right] \tau}\right]^{-1} .
$$

We use a numerical search algorithm to determine the radiant exposure, $H_{\mathrm{th}}$, that satisfies this equation.

\section{Results}

Measurements for $Z_{1}$ and $E_{a 1}$ exist in the literature, but no such measurements exist for $Z_{2}$ and $E_{a 2}$. However, we can use the 


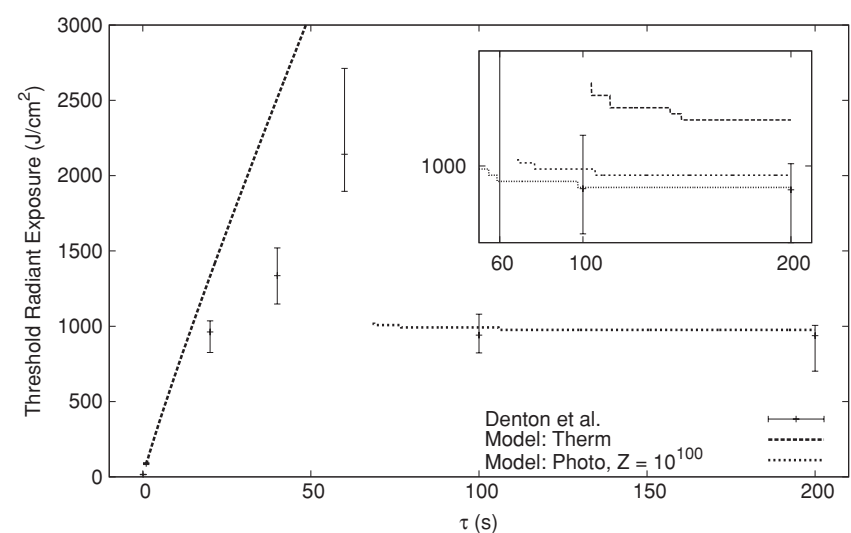

Fig. 2 Predicted temporal action profile (line) compared to the measured damage thresholds by Denton et al. ${ }^{9}$ (points). The inset shows photochemical damage predictions for three different collision frequencies: $10^{80}$ (top), $10^{100}$ (middle), and $10^{120}$ (bottom).

critical temperature (the temperature for which the Arrhenius rate is equal to one $\left.{ }^{11}\right), T_{c}=E_{a 2} / R \ln Z_{2}$, to define the relationship between $Z_{2}$ and $E_{a 2}$. We previously showed ${ }^{9}$ that the expected temperature for the $100 \mathrm{~s}$ threshold exposure was around $38^{\circ} \mathrm{C}$, while the expected temperature for the $60 \mathrm{~s}$ threshold exposure was about $48^{\circ} \mathrm{C}$. This is in-line with the rule of thumb that thermal damage requires about a $10^{\circ} \mathrm{C}$ temperature rise. Here, we assume that the critical temperature associated with the photochemical rate process is $47^{\circ} \mathrm{C}(320.15 \mathrm{~K})$.

We use the thermal damage rate coefficients reported by Welch and Polhamus for thermal damage: ${ }^{12} E_{a 1}=6.28 \times 10^{5} \mathrm{~J}$ $\mathrm{mol}^{-1}, Z_{1}=3.1 \times 10^{99} \mathrm{~s}^{-1}$. The expected temperature rises reported by Denton et al. ${ }^{9}$ give $m=0.26$, and the reciprocity energy was $940 \mathrm{~J} \mathrm{~cm}^{-2}$. We have computed damage threshold predictions for three different values of $Z_{2}: 10^{80}, 10^{100}$, and $10^{120}$. With $E_{a 2}=320.15 R \ln Z_{2}$, this gives $4.9 \times 10^{5}, 6.13 \times 10^{5}$, and $7.35 \times 10^{5}$ for $E_{a 2}$. The model predicts the same sharp transition from thermal to photochemical damage as was observed (Fig. 2), but the transition region depends on the value of $Z_{2}$. The smallest collision frequency $\left(Z_{2}=10^{80}\right)$ gives photochemical damage at exposure duration less than $60 \mathrm{~s}$, while the largest $\left(Z_{2}=10^{120}\right)$ leads to no photochemical damage at $100 \mathrm{~s}$. The best agreement was with $Z_{2}=10^{100}$.

\section{Conclusions}

We have presented a theoretical model that assumes a temperature dependent photochemical damage rate and provides an explanation for the sharp transition between thermal and photochemical damage recently observed in in-vitro and in-vivo data for short wavelength damage thresholds. As the exposure duration is decreased, the irradiance must be increased to deliver the same number of photons. But the increase in irradiance leads to a larger temperature rise, which eventually disables the photochemical damage. We have not attempted to identify the underlying pathways responsible for causing photochemical damage; the model only assumes that the damage rate can be described by a rate equation. A discussion of possible photochemical mechanisms at the molecular level can be found in Ref. 13.

The damage threshold predictions reproduce the sharp transition between thermal and photochemical damage observed in the in-vitro data reported by Denton et al. ${ }^{9}$ The two rate coefficients for photochemical damage quenching have been estimated, but these would need to be determined experimentally to make quantitative predictions. In principle, these coefficients would need to be determined for each tissue and endpoint to be described, but could then be used to predict whether or not photochemical damage is caused under various exposure conditions, similar to how the thermal damage Arrhenius coefficients are used today.

\section{References}

1. F. C. Henriques, "Studies of thermal injury $\mathrm{v}$ the predictability and the significance of thermally induced rate process leading to irreversible epidermal injury," Arch. Pathol. 43, 489-502 (1947).

2. M. A. Mainster, T. J. While, J. H. Tips, and P. W. Wilson, "Transient thermal behavior in biological systems," Bull. Math. Biol. 32, 302-315 (1970).

3. A. M. Clarke, W. J. Geeraets, and W. T. Ham Jr., "An equilibrium thermal model for retinal injury from optical sources," Appl. Opt. 8(5), 1051-1054 (1969).

4. A. N. Takata, L. Zaneveld, and W. Richter, "Laser-induced thermal damage of skin," Technical report, IIT Research Institute (1977).

5. B. Chen, "Histological and modeling study of skin thermal injury to 2.0 micron laser irradiation," Lasers Surg. Med. 40, 358-370 (2007).

6. Y. Feng, J. T. Oden, and M. N. Rylande, "A two-state cell damage model under hyperthermic conditions: theory and in vitro experiments," J. Biomech. Eng. 130, 041016 (2008).

7. S. J. Till, J. Till, P. K. Milsom and G. Rowlands, "A new model for laserinduced thermal damage in the retina," Bull. Math. Biol. 65, 731-746 (2003).

8. J. Wu, S. Seregard, and P. V. Algvere, "Photochemical damage of the retina," Surv. Ophthalmol. 51, 461-481 (2006).

9. M. L. Denton, C. D. Clark III, M. S. Foltz, K. J. Schuster, G. D. Noojin, L. E. Estlack, and R. J. Thomas, "In vitro retinal model reveals a sharp transition between laser damage mechanisms," J. Biomed. Opt. 15, 030532 (2010).

10. D. J. Lund, B. Stuck, and P. Edsall, "Retinal injury threshold for blue wavelength lasers," Health Phys. 90, 477-484 (2006).

11. A. Welch and M. van Germert, Optical-Thermal Response of LaserIrradiated Tissue, Plenum Press, New York (1995).

12. A. Welch and G. Polhamus, "Measurement and prediction of thermal injury in the retina of the rhesus monkey," IEEE Trans. Biomed. Eng. 31, 633-644 (1984).

13. B. S. Gerstman and R. D. Glickman, "Activated rate processes and a specific biochemical mechanism for explaining delayed laser induced thermal damage to the retina," J. Biomed. Opt. 4, 345-351 (1999). 